\title{
Does the Incredible Years Teacher Classroom Management Programme Improve Child-Teacher Relationships in Childcare Centres? A One-Year Universal Intervention in a Norwegian Community Sample
}

Håvard Horndalen Tveit ${ }^{1}$, May Britt Drugli ${ }^{1,2}$, Sturla Fossum ${ }^{3}$, Bjørn Helge Handegård ${ }^{3}$, Frode Stenseng ${ }^{4,5}$

${ }^{1}$ The Regional Centre for Child and Youth Mental Health and Child Welfare - Central Norway, Norwegian University of Science and Technology (NTNU), Trondheim, Norway

${ }^{2}$ Centre for the Study of Educational Practice (SePu), Hedmark University College, Elverum, Norway

${ }^{3}$ The Regional Centre for Child and Youth Mental Health and Child Welfare - North, UiT, The Arctic University of Norway, Tromsø, Norway

${ }^{4}$ Department of Education and Lifelong Learning, Norwegian University of Science and Technology (NTNU), Trondheim, Norway

${ }^{5}$ Queen Maud University College of Early Childhood Education (DMMH), Trondheim, Norway

Corresponding author: Håvard Horndalen Tveit, tel.: +47 482 62424, email: havard.tveit@ntnu.no.

ORCID: 0000-0003-3262-761X 


\begin{abstract}
The Incredible Years Teacher Classroom Management (IY TCM) programme has shown promise in reducing behaviour problems among high-risk children in childcare. However, at present, we do not know whether the IY TCM successfully improves the child-teacher relationship in childcare and whether the effects manifest in both the population and in high-risk groups. Hence, we conducted a quasi-experimental pre-post study with a matched control condition to examine the changes in child-teacher relationships in a sample of 1085 children aged 3-6 years after implementing the IY TCM programme. Linear mixed models revealed favourable group-by-time differences benefitting the intervention compared to the control condition. Subgroup analyses of children scoring at or above the 90th percentile on either internalising or externalising behaviour problems showed that the preventive effects persisted in both high-risk subsamples. In sum, the findings indicate that the IY TCM programme does improve child-teacher relationships and that the effect is present for the entire study population as well as children scoring in the clinical range on behaviour problems. This suggests that the application of the IY TCM programme in childcare settings has important preventive effects. Implications and limitations are further discussed.
\end{abstract}

Keywords: child-teacher relationship, intervention, incredible years, internalising, externalising, childcare 
Does the Incredible Years Teacher Classroom Management Programme Improve Child-Teacher Relationships in Childcare Centres? A One-Year Universal Intervention in a Norwegian Community Sample

\section{Introduction}

The detrimental legacy of mental health problems in early childhood is profound and includes adolescent and adult academic problems, crime, substance abuse, unemployment, and poor quality of life [1-4]. Hence, the development and implementation of procedures to prevent or reduce such early mental health problems is pivotal $[5,6]$. Several school- and childcare-based primary prevention efforts have proven effective in this respect [7-9], including the Incredible Years Teacher Classroom Management (IY TCM) [10], which is the focus of the present study. The IY TCM aims, as the name suggests, to alter teacher classroom management and thereby prevent both internalising and externalising behaviour [10]. Indeed, several intervention studies indicate that the IY TCM is successful in this regard and report moderate to high preventive effects on measures of conduct problems, self-regulation, and social competence in high-risk populations [10,11]. From a developmental and observational line of research, one particular factor has been identified as a buffer against later mental health problems and externalizing behaviour in particular, namely, a positive child-teacher relationship, characterised by trust, warmth, and little conflict [12]. However, the extent to which one of the most promising intervention efforts, the IY TCM, alters what is potentially the most important factor in mental health problems, the child-teacher relationship, is currently not known for children in childcare; thus, it is the primary focus of the present study.

\section{The Importance of the Child-Teacher Relationship}

According to attachment theory [13], the relationship between a child and his or her parents is essential for the child's development; secure and stable relationships may facilitate resilience against risk factors, whereas the lack thereof may place children on less adaptive developmental trajectories [12]. Scholars have argued for the concordance of parents' and teachers' ability to provide stable bonds and thus decrease the risk for adjustment problems [14], a proposition supported in a large-scale meta-analysis [15]. Furthermore, in cases where the parental setting may be a risk factor itself, adult caregivers' sensitivity to the child may to some extent amend the child's prior attachment relationship experiences and form new, healthy, and protective attachments [16]. Indeed, a recent meta-analysis concluded that a positive child-teacher relationship, in particular one with little conflict, is associated 
with fewer mental health problems and behaviour problems in general [17]. These cross-sectional observations are corroborated by a range of prospective studies strongly suggestive of the importance of child-teacher conflict for later emotional and behaviour problems $[12,18]$. Thus, for children who display or are at risk of developing mental health problems, positive relationships with teachers seem to be particularly important as a protective factor [19,20]. The reciprocal influence between teacher and child indicates that conflictual relationships may be reinforced when left untreated, further affecting children's subsequent development and adjustment [21,22]. In sum, existing knowledge firmly suggests that a positive child-teacher relationship in childcare protects against later emotional and behavioural problems. How to improve this relationship, however, is less researched.

\section{The Incredible Years Teacher Classroom Management (IY TCM) Programme}

The Incredible Years (IY) Series is a set of preventive intervention programmes for children, parents and teachers aimed at promoting socioemotional competence and preventing, reducing and treating conduct problems among young children [23]. The programmes make use of detailed instructional manuals, demonstrational video vignettes, reading assignments, collaborative workshops with group discussions led by IY instructors, and checklists to promote fidelity. The teacher module, the Incredible Years Teacher Classroom Management (IY TCM) programme, is firmly grounded in attachment and social learning theory, identifying the role of the teacher as not only an educator but as the child's primary caregiver in the school and childcare setting [24]. The relationship between child and teacher takes precedence over pedagogy, and classroom management entails strengthening the child-teacher bond and cultivating behaviour management, emotional regulation, and social skills [10]. In a previous study [25], we reported reductions in internalising and externalising behaviour problems in addition to increased social competence in childcare centres that implemented the IY TCM programme over one year. Furthermore, moderate to large intervention effects have been documented with regard to reductions in conduct problems and disruptive behaviour [26-29] and positive improvements in classroom management [28-31], social skills [26,27] and teacher sensitivity [28]. The use of positive classroom strategies could greatly affect the relationships between teachers and children, increasing levels of closeness and decreasing levels of conflict; however, this interaction has not yet been examined in a childcare setting. As the prime purpose of IY TCM is to prevent and reduce emotional and behavioural problems, it is particularly important to investigate whether the programme improves child-teacher 
relationships not only for the general population but also among children displaying initial signs of emotional and behavioural problems.

\section{The Present Study}

In the present study, which used a quasi-experimental pre-post design with a matched control conditions comprising 1085 children aged 3 to 6 years, we hypothesized the following:

(1) After participating in the IY TCM programme, childcare teachers would report more favourable changes in closeness and conflict in their child-teacher relationships relative to nonparticipating teachers in the matched control condition.

(2) Children in the intervention condition with increased levels of internalising or externalising problems would experience the same favourable changes as the entire sample compared to high-risk children in the matched control condition.

\section{Methods}

\section{Participants}

In Norway, $97.1 \%$ of all children ages three through five attend childcare centres, and $96.4 \%$ of the children spend 41 hours or more per week at their unit [32]. Attendance among children from linguistic and cultural minorities are high (82.2\%), and the government ensures affordable childcare for all through national regulations, regardless of public or private ownership, with low-income families receiving partial funding for childcare [32]. All childcare centres in Norway has to act in accordance to the Kindergarten Act [33], stating there must be at least one caregiver per six children over the age of three, at least one pedagogical leader per 14-18 children, and pedagogical leaders must be educated pre-school teachers or possess comparable qualifications. Furthermore, the Framework Plan provides guidelines for values, content, learning objectives and tasks each childcare centre must implement in their unit [33]. Finally, though not stated explicitly in any regulations, most childcare centres have a keyworker system, with one member of staff having particular responsibility for each child at the unit.

Municipalities that had previously implemented the Incredible Years Parenting Programme were invited to receive implementation of the Teacher Classroom Management programme in their childcare units. These 
municipalities were chosen because they had available group leaders who could easily be trained in the IY TCM programme. Childcare centres that wanted to participate were required to apply to IY Norway and received the implementation free of charge in addition to a modest financial compensation for completing the questionnaires. To be eligible for inclusion in the intervention condition, all units in the childcare centres had to participate in the programme, at least $80 \%$ of the childcare staff had to approve of implementation, and the staff were prohibited from attending or participating in any other educational training or behavioural programmes during the study period. Forty-six childcare centres applied and were included in the intervention condition, which comprised 1527 children.

Childcare centres from municipalities where none of the IY programmes were currently available were invited to apply to IY Norway for participation in the control condition. These units were strategically matched to the intervention centres according to geographical location (rural versus urban) and the number of children enrolled. Similar to the intervention condition, childcare centres in the control condition received the same financial compensation and were offered the opportunity to implement the IY TCM programme one year later. The matched control condition likewise consisted of forty-six childcare centres including 1634 children.

To reduce the childcare teachers' burden of completing the assessment and to limit data dependency (see statistical analysis for further description), seven children in each childcare unit were randomly selected to be included in the study. The procedure consisted of generating a random number list equal to the number of children in the unit, which the childcare teacher matched with the alphabetical list of children in attendance. The first seven children on the random number list would then be included in the study. The procedure resulted in 581 children in the intervention condition and 637 children in the control condition. After the allocation, but before the study began, one childcare centre in the intervention condition, including 51 children, and two childcare centres in the control condition, including 82 children, dropped out due to organisational matters unrelated to the intervention. Thus, the final sample included 530 children from 45 childcare centres in the intervention condition, and 535 children from 44 childcare centres in the control condition.

Furthermore, subgroup analyses were conducted on subsamples of children with an elevated risk of internalising or externalising behaviour problems as indicated by the Caregiver-Teacher Report Form (C-TRF). The optimal cut-off point for discrimination between clinical and nonclinical children has been demonstrated at the 90th percentile in normative samples [34]; for this reason, we used this threshold in our secondary analyses. As the CTRF scales are measured in integer units, the subsamples included all participants with index scores equal to the 
90th percentile score. The 90th percentile scores for internalising and externalising behaviour problems were 8 and 15 , respectively, and included $10.9 \%$ and $11.0 \%$ of the sample. Due to an error with the questionnaires for the first 215 participants (137 in the intervention and 78 in the control condition), only the internalising domain of the CTRF were included in the baseline assessment; thus, the high-risk subsample is based only on the participants with valid data at baseline. The subsample of children at high risk for internalising behaviours consisted of 47 participants in the intervention condition and 48 in the control condition, whereas the externalising subsample included 46 participants in the intervention condition and 50 in the control condition.

\section{Procedures}

The study was structured as a quasi-experimental, pre-post design with a matched control condition. Enrolment of childcare centres in the intervention and control conditions was continuous over the five year study period, from fall 2009 to fall 2013. Before commencement, the procedures and study protocol were approved by the Regional Committee for Medical and Medicine Research Ethics (REK Nord). Information regarding the IY TCM programme and the research study was verbally presented to the childcare staff; the parents were informed about the study during parent meetings in addition to receiving written information. As written information and consent forms were only available in Norwegian, children of parents with insufficient proficiency in Norwegian were excluded. Since all childcare teachers partook in the IY TCM training, all children in the unit would be affected by the intervention, but only children whose parents consented would be eligible for assessment. After receiving written parental consent, the childcare teachers completed the questionnaires. The baseline assessment was conducted one to three weeks ahead of the first IY TCM workshop, and the follow-up assessment was conducted one to three weeks after the final workshop. Both assessments included the measures of child-teacher relationship (STRS-SF), as well as measures of internalising and externalising behaviour problems (C-TRF). The period between assessments was eight to nine months.

Sample size estimations to detect an effect size of .20 (Hedges' g) at .05 alpha and .80 power for two-sided comparison of the intervention and control condition on the measures of child-teacher relationship resulted in 394 participants. As the location of dependency was expected at the teacher level, we adjusted the sample size for the design effect [35], based on the degree of dependence measured by the intraclass correlation coefficient (ICC) and the relative cost of level-1 and level-2 sampling. A conservative ICC of .20 was assumed, in line with common 
recommendations for behavioural outcome measures [36], and the relative cost of sampling at each level was determined by balancing the lowest possible design effects, the workload on the teachers, and the unique contribution of each participant at the level-1 unit. The final sample size needed was 867 children (level-1 sampling size) at 124 childcare centres (level-2 sampling size), with a design effect of 2.2. As the ICC and cluster size can dramatically influence the power of studies with clustered data, we calculated the precise ICC after two years of data collection, and re-estimated the robust sample size [37], which prompted us to stop collecting data after 46 childcare centres were included in both conditions.

\section{The Incredible Years Teacher Classroom Management (IY TCM) Programme}

The IY TCM programme is a universal preventive intervention for children ages three to eight years; it is intended for full implementation in all units at schools or childcare centres involved [10]. Designed as a flexible programme that maintains high fidelity, the intervention is a result of reciprocal interaction between trained group leaders with knowledge of content and principles and participating teachers with knowledge of the children and the social context.

The programme is structured around six full-day workshops for all childcare staff involved with the children at each childcare centre and is led by two experienced and qualified IY TCM group leaders. Each workshop lasts approximately 6-7 hours, is held every fourth week over an eight- to nine-month period, and revolves around specific topics pertinent to the childcare setting, as instructed in the accompanying manual. The topics for the six workshops are (i) building a positive relationship with the child and preventing behaviour problems; (ii) the importance of teacher attention, coaching and praise; (iii) motivating children through incentives; (iv) ignoring and redirecting inappropriate behaviour among children; (v) following through with consequences; and (vi) developing children's emotional regulation, social skills, and problem solving.

The childcare staff participated in lectures, discussions, role-play, and reading assignments based on the topics covered and were instructed to practice the principles during the following month and report on their experiences at the start of the next workshop. In line with the manual, the group leaders provided either verbal or written guidance between the workshops [38]. To promote fidelity in implementation, in addition to following the manual, checklists were completed by both the childcare staff and the group leaders after each workshop, and a user satisfaction questionnaire was administered. 
The 20 group leaders who provided IY TCM in this study worked in Educational-Psychological Services and were trained educators with higher education qualifications in teaching, special education, psychology or social studies. They followed a 21-hour IY TCM training course provided by IY Norway and had to deliver the programme to at least one or two childcare centres or schools prior to becoming group leaders. All group leaders involved in the study were trained and supervised by the same two IY TCM mentors (certified in both the Parenting and Teacher programmes by the programme originator).

\section{Measures}

\section{Child-Teacher Relationship}

The teacher's perceptions of their relationship with a particular child were measured with the Student-Teacher Relationship Scale Short Form (STRS-SF) [39]. The STRS-SF is a self-report questionnaire composed of 15 items evaluated on a five-point Likert scale, where a score of $1=$ "Definitely does not apply" and a score of $5=$ "Definitely does apply". The scale encompasses two divergent relationship dimensions: closeness (seven items), measuring the degree to which the relationship is recognized as affectionate and warm (e.g., "It is easy to be in tune with what this child is feeling"), and conflict (eight items), measuring the degree to which the relationship is perceived as conflictual and cold (e.g., "This child and I always seem to be struggling with each other"). The closeness scale ranges from 7-35, with higher scores reflecting warmer relationships, while the conflict scale ranges from 8-40, with higher scores indicating more negative relationships. Both concurrent and factor validity have previously been documented in a Norwegian setting [40] (with results favouring the short form rather than the original), and the test-retest reliability at a four-week interval was .88 for closeness and .92 for conflict [39]. For the age-range studied, normative scores have been reported as 27.13 for closeness and 11.62 for conflict [41]. In the present study, the internal consistency was .80 and .78 for closeness and .80 and .81 for conflict at baseline and follow-up, respectively.

\section{Child's Behavioural, Emotional, and Social Functioning}

The Caregiver-Teacher Report Form (C-TRF) [34] was used to examine internalising and externalising problems. With the C-TRF, the teachers rate the occurrences of potentially problematic behaviour for each child on a 99-item 
questionnaire ranging from $0=$ "Not true (as far as you know)" to $2=$ "Very true or often true". To obtain a child's unique score on the internalising scale, one must sum the scores from the following four subscales: emotionally reactive (seven items), anxious/depressed (eight items), somatic complaints (seven items), and withdrawn (10 items). Likewise, the externalising scale corresponds to the sum of the subscales attention problems (nine items) and aggressive behaviour (25 items). The validity and reliability of the C-TRF has been extensively examined in previous studies [34]. For the age range measured in the current study, comparable population means for the C-TRF comes from Denmark, with a 4.2 index score on internalising and 4.1 at externalising [42]. The internal consistency of the subscales was estimated due to the multidimensionality of the tests; for the internalising measures, internal consistency was .66 and .63 for emotionally reactive, .60 and .62 for anxious/depressed, .45 and .34 for somatic complaints, and .80 and .74 for withdrawn at baseline and follow-up, respectively. For the externalising problems measures, internal consistency was .87 and .86 for attention problems and .93 and .93 for aggressive behaviour.

\section{Demographical Data}

Factors that were expected to potentially influence the analyses of the study were collected beforehand. These included the number of children in each condition, sex and age and are presented in Table 1.

\section{Statistical Analyses}

Demographical variables were analysed to examine any differences between the intervention and control conditions and between the completers and those lost to follow-up using independent samples $t$-test, Pearson's chi-square or linear mixed models. Linear mixed models were applied to test for group differences at baseline and group-by-time effects in the STRS-SF scores between baseline and follow-up. Accounting for the hierarchical structure of the data, repeated observations were nested within children, and children were nested within teachers for the analyses of the

entire sample, whereas the teacher level was dropped from the subgroup analyses as few teachers had more than one high-risk participant in her or his unit. As some of the children were included in both subgroup analyses, an indicator was included in the analyses to correct for comorbidity.

In the case of missing responses on either the STRS-SF or the C-TRF, the standard procedures described in the respective manuals were followed. For the STRS-SF [39], the accepted number of missing items is one per subscale, in which case the missing value is replaced with the mean score of the available items within the subscale 
being calculated. The C-TRF manual [34] requires that no more than eight of the total 99 items are missing, and if so, that the same calculation procedure as utilised for the STRS-SF is followed. If more than the accepted number of items was missing, the subscale was determined invalid and left out of the analysis.

The results are reported as estimated marginal means with standard errors based on the linear mixed models. All observations were included as missing data at follow-up and were assumed to be missing at random, in which case linear mixed models with full maximum likelihood estimation should provide precise estimates of the effects nonetheless. Effect sizes were computed as the estimate of the group-by-time effect divided by the pooled standard deviation at baseline [43]. The ICC was estimated from baseline with linear mixed models using the unconditional means model [44]. A two-sided $p$-value of less than .05 was considered statistically significant in every test.

\section{Results}

A total of 1085 children were included in the data analyses: 530 in the intervention and 555 in the control condition. A total of 921 children (435 in the intervention condition, 486 in the control condition) had complete data at both timepoints, and 121 children were lost to follow-up. Drop-out was due to lack or responses from three childcare centres (24 in the intervention condition, 15 in the control condition; childcare teacher drop-outs resulted in missing responses from 19 children in the intervention condition and 5 in the control condition; and finally, there were 29 child drop-outs in each condition. Furthermore, another 43 children, 23 in the intervention condition and 20 in the control condition, were included in the follow-up assessment from three childcare centres (two in the intervention condition and one in the control condition) that participated in the study but did not complete the baseline assessment (see Fig. 1). We tested for the group-by-attrition interaction and found that compared to the children in the control condition with data at both assessments, the children in the control group who were lost to follow-up scored lower on STRS-SF Closeness ( -1.92 index points; $95 \%$ CI: -3.21 to $-0.65 ; p=.003)$, but higher on STRS-SF Conflict (1.67 index points; 95\% CI: 0.11 to $3.22 ; p=.036$ ) and C-TRF Internalising (1.81 index points; 95\% CI: 0.48 to $3.12 ; p=.008)$. No other group-by-attrition interaction was significant.

Analyses of the baseline characteristics showed no difference between the intervention and control conditions on any measures (Tab. 1). In line with hypothesis 1 , the childcare teachers in the intervention condition reported more favourable changes in STRS-SF Closeness (1.21 index points; $95 \%$ CI: 0.78 to $1.65 ; p<0.001)$ and Conflict $(-0.97$ index points; $95 \%$ CI: -1.49 to $-0.46 ; p<0.001)$ in their child-teacher relationships than teachers in 
the matched control condition (Tab. 2). The subgroup analyses of the Internalising subsample showed the preventive effects persisted in favour of the intervention condition on STRS-SF Closeness ( 2.24 index points; $95 \%$ CI: 0.69 to 3.78; $p=0.006)$ and Conflict ( -2.33 index points; $95 \%$ CI: -4.33 to $-0.34 ; p=0.024)$. Likewise, the Externalising subsample in the intervention condition revealed favourable improvements on STRS-SF Closeness (1.56 index points; $95 \%$ CI: 0.69 to $3.78 ; p=0.033)$ and Conflict $(-2.93$ index points; $95 \%$ CI: -5.15 to $-0.71 ; p=0.011)$.

\section{Discussion}

As a positive child-teacher relationship has been identified as arguably the strongest protective factor in the childcare setting against later child mental health problems, we examined whether the Incredible Years Teacher Classroom Management (IY TCM) [10] programme could improve child-teacher relationship in childcare centres during a nine-month period. We hypothesised that, first, teachers in the intervention condition would report more favourable changes in closeness and conflict in their child-teacher relationships relative to nonparticipating teachers in the matched control condition; this proposition was supported by the results. Second, we examined whether children with increased levels of internalising or externalising behaviour problems would experience the same favourable changes as the entire sample, a contention that was also supported by the data. The preventive effects for the entire sample would be classified as small by Cohens criteria of effect size interpretation [45], which is to be expected given the high levels of closeness and low levels of conflict present at baseline between the childcare teachers and the children. The subgroup analyses revealed moderate preventive effects for both measures, with estimated follow-up scores close to the population scores on the closeness dimension and conflict scores substantially lower than at baseline, which is particularly promising given the potential benefits for high-risk children.

Although the quality of relationships with primary caregivers outside of the home has been the focus of much theoretical and empirical research over the last 20 years, few interventions have aimed at improving this facet in the childcare setting [14]. Our findings demonstrate that child-teacher relationships are indeed malleable over the course of a nine-month universal preventive intervention programme delivered in childcare centres for both the entire study sample and those with elevated levels of internalising or externalising behaviour problems. The population approach to prevention postulates that universal measures are more beneficial than exclusively targeting those with an elevated risk of adverse outcomes [46]. Although the incidence ratio is greater in high-risk groups, the 
total incidence rate is higher in the general population; consequently, shifting the risk distribution by even a small amount for the entire population will result in greater public health gains [47]. Our study fits well with these predictions, revealing small effects in the study population and moderate gains among the high-risk subsamples, corroborating the population approach's benefit as a means of preventing and reducing new incidences. As previously documented [48], children with increased levels of emotional or behaviour problems, particularly children with externalising behaviour, have more conflictual relationships with their teachers. Given the reciprocal effects of conflictual relationships [22] and the way that child-teacher relationships in childcare often carry over to school [49], the benefits of lower levels of conflict could have lasting positive outcomes for these groups, making the transition from childcare to school easier for both the children and their parents. Children with internalising behaviour problems may also suffer in groups consisting of children with externalising problems, as externalising behaviour tends to overshadow other behaviours and demand more attention. Further reasons to support treating the entire unit come from a recent study showing that bidirectional influences in child-child relationships lead to the codevelopment of traits, reinforcing both negative and positive characteristics and making groups more homogenous over time [50]. This group reinforcement mechanism implies that if left untreated, children scoring in the upper end of the behaviour problem scales may pull the lower-scoring children towards them on the scale and influence the classroom environment in a negative manner. Likewise, nurturing stronger and healthier relationships between children and teachers in the entire unit may cause the children to centre around lower levels of internalising and externalising behaviour.

An enduring question is whether the preventive effects of the IY TCM programme we have documented will persist, increase or decrease over time. Studies on the long-term effects of the IY parent module [51] and the combined parent and teacher module [52] show promise, documenting lasting effects up to 5-6 years after the intervention. One of the strengths of the programme is its focus on training the teachers, which ensures that the necessary skills are embedded in the teachers and thus become part of the teacher's pedagogical repertoire after the intervention period is over. Indeed, the continued use of the proactive teaching techniques acquired through the IY TCM intervention has been confirmed in previous studies, both in settings where the teachers independently incorporated these strategies [30] and in settings where the teachers received weekly visits from mental health consultants [53]. To maintain these techniques over time, teacher training alone may not be sufficient [54], but the comprehensiveness of the IY TCM programme, which includes lectures, discussions, role-play, reading assignments, 
and guidance, may help the teachers grasp the mechanisms more fully and help keep them in active use [31]. Finally, it would be interesting to examine whether the ability to create meaningful child-teacher relationships are confined to the teachers, or whether the children themselves understand how these social bonds are formed and maintained. A substantial part of the sample will transition to school quite soon, and following these children into this new arena could greatly elaborate these questions in future studies. The link between child-teacher relationships and academic achievement and absenteeism could also be examined furthered, and it may be that we have not yet understood all the potential preventive effects the IY TCM can have on children.

\section{Limitations}

This study has some limitations. First, the current study was not a randomised control trial; it was conducted in a natural setting with pre-post assessments and a nonrandomly matched control condition. Consequently, caution is warranted when interpreting the results, particularly in terms of unmeasured confounding variables, and in the subgroup analyses, regression to the mean [55]. However, as Shadish et al. [56] argue, the pre-post design with a matched control condition is among the soundest designs and is most likely to permit causal interpretations as far as quasi-experimental design goes. Second, the sampling procedure relied on childcare centres that applied to IY Norway for participation, an approach that is vulnerable to validity threats. Willingness to participate in any type of intervention could influence the results, but as would unwillingness to participate, and willingness to participation must be a given for conducting any intervention. It would have been informative to have data on both childcare centres who applied and those who chose not apply, but as data could not be collected from childcare centres who did not participate in the trial, we had no grounds for comparison. To address these methodological weaknesses, we examined the baseline differences between the intervention and control conditions and between children lost to follow-up versus children with data at both timepoints and chose appropriate statistical methods that account for these discrepancies. Third, the assessments were completed exclusively by the childcare teachers and may reflect their perception of what constitutes problems rather than accurately reflecting the children's behavioural problems. Thus, the causal predictor of change in the study may not be the intervention itself; rather, it may be a deeper understanding of child behaviour following the IY TCM modules, which led the teachers to make more precise observations at the follow-up assessment. There is also a risk of response bias, as teachers having undergone training might respond more favourably not as a result of the intervention, but rather as a result of the belief in the training or 
wanting to show they have improved, but this is a difficult bias to overcome in prevention research. One may argue the study would benefit from adding observational data from the childcare centres, with trained observers blinded to the condition to amend potential response bias. Unfortunately, this was not possible due to the financial burden, and would have forced us to collect a substantially lower level-2 sample size. Furthermore, as training and implementation were carried out by IY Norway, we had limited control over the fidelity of the intervention, and it is possible that the fidelity varied between childcare centres. However, if this is the case, it would be expected that the preventive effects found in our data are underestimated, not overestimated, and that higher levels of fidelity would result in greater intervention effects [57]. We do recommend that future studies include measures of fidelity. Finally, the high-risk subsample sizes were relatively small, and the results in this situation should therefore be interpreted with caution.

\section{Conclusion}

Our data indicate that delivering the IY TCM as a universal preventive intervention for children in childcare may improve one of the prime teacher-related protectors against child mental health problems: the quality of the childteacher relationship. Although our results are in need of replication, the positive effect of the IY TCM on relationship quality appears to affect the study population as a group as well as those children who scored in the clinical range. These findings corroborate previous studies on the preventive effects of IY TCM and suggest that it might be a suitable preventive tool in childcare centres.

\section{Conflict of Interest}

The authors declare that they have no conflict of interest.

\section{Ethical Approval}

All procedures performed in studies involving human participants were in accordance with the ethical standards of the institutional and/or national research committee and with the 1964 Helsinki declaration and its later amendments or comparable ethical standards.

\section{Informed Consent}


Informed consent was obtained from all individual participants included in the study.

\section{Acknowledgements.}

The authors wish to thank Professor Willy-Tore Mørch for his contribution to the study design, Anne-Kari Johnsen, Merete Aasheim and Oddbjørn Løndal for acquisition of data, parents for their willingness to participate, and the childcare teachers for completing the assessments. 


\section{References}

1. Layard R, Clark A, Cornaglia F, Powdthavee N, Vernoit J (2014) What predicts a successful life? A lifecourse model of well-being. Econ J 124:F720-F738. https://doi.org/10.1111/ecoj.12170

2. Odgers CL, Moffitt TE, Broadbent JM, Dickson N, Hancox RJ, Harrington H, Poulton R, Sears MR, Thomson WM, Caspi A (2008) Female and male antisocial trajectories: from childhood origins to adult outcomes. Dev Psychopathol 20:673-716. https://doi.org/10.1017/S0954579408000333

3. Shaw DS, Gilliom M, Ingoldsby EM, Nagin DS (2003) Trajectories leading to school-age conduct problems. Dev Psychol 39:189-200. https://doi.org/10.1037/0012-1649.39.2.189

4. Fergusson DM, Horwood LJ, Ridder EM (2005) Show me the child at seven: the consequences of conduct problems in childhood for psychosocial functioning in adulthood. J Child Psychol Psychiatry 46:837-849. https://doi.org/10.1111/j.1469-7610.2004.00387.x

5. Masten AS, Cicchetti D (2010) Developmental cascades. Dev Psychopathol 22:491-495. https://doi.org/10.1017/S0954579410000222

6. Heckman JJ (2006) Skill formation and the economics of investing in disadvantaged children. Science 312:1900-1902. https://doi.org/10.1126/science.1128898

7. Sanders MR (1999) Triple P-positive parenting program: towards an empirically validated multilevel parenting and family support strategy for the prevention of behavior and emotional problems in children. Clin Child Fam Psychol Rev 2:71-90. https://doi.org/10.1023/A:1021843613840

8. August GJ, Realmuto GM, Hektner JM, Bloomquist ML (2001) An integrated components preventive intervention for aggressive elementary school children: the early risers program. J Consult Clin Psychol 69:614-626. https://doi.org/10.1037/0022-006X.69.4.614

9. Schweinhart LJ, Weikart DP (1997) The high/scope preschool curriculum comparison study through age 23 . Early Child Res Q 12:117-143. https://doi.org/10.1016/S0885-2006(97)90009-0

10. Webster-Stratton C, Reinke WM, Herman KC, Newcomer LL (2011) The Incredible Years teacher classroom management training: the methods and principles that support fidelity of training delivery. Sch Psych Rev 40:509-529

11. Pidano AE, Allen AR (2015) The Incredible Years series: a review of the independent research base. J Child Fam Stud 24:1898-1916. https://doi.org/10.1007/s10826-014-9991-7 
12. Sabol TJ, Pianta RC (2012) Recent trends in research on teacher-child relationships. Attach Hum Dev 14:213-231. https://doi.org/10.1080/14616734.2012.672262

13. Ainsworth MDS, Blehar MC, Waters E, Wall SN (1978) Patterns of attachment: a psychological study of the strange situation. Erlbaum, Hillsdale, NJ

14. Verschueren K, Koomen HM (2012) Teacher-child relationships from an attachment perspective. Attach Hum Dev 14:205-211. https://doi.org/10.1080/14616734.2012.672260

15. Ahnert L, Pinquart M, Lamb ME (2006) Security of children's relationships with nonparental care providers: a meta-analysis. Child Dev 77:664-679. https://doi.org/10.1111/j.1467-8624.2006.00896.x

16. Buyse E, Verschueren K, Doumen S (2011) Preschoolers' attachment to mother and risk for adjustment problems in kindergarten: can teachers make a difference? Soc Dev 20:33-50. https://doi.org/10.1111/j.1467-9507.2009.00555.x

17. Cornelius-White J (2007) Learner-centered teacher-student relationships are effective: a meta-analysis. Rev Educ Res 77:113-143. https://doi.org/10.3102/003465430298563

18. McGrath KF, Van Bergen P (2015) Who, when, why and to what end? Students at risk of negative studentteacher relationships and their outcomes. Educ Res Rev 14:1-17. https://doi.org/10.1016/j.edurev.2014.12.001

19. Hamre BK, Pianta RC (2005) Can instructional and emotional support in the first-grade classroom make a difference for children at risk of school failure? Child Dev 76:949-967. https://doi.org/10.1111/j.14678624.2005.00889.x

20. Ladd GW, Burgess KB (2001) Do relational risks and protective factors moderate the linkages between childhood aggression and early psychological and school adjustment? Child Dev 72:1579-1601. https://doi.org/10.1111/1467-8624.00366

21. Buyse E, Verschueren K, Doumen S, Van Damme J, Maes F (2008) Classroom problem behavior and teacher-child relationships in kindergarten: the moderating role of classroom climate. J Sch Psychol 46:367-391. https://doi.org/10.1016/j.jsp.2007.06.009

22. Doumen S, Verschueren K, Buyse E, Germeijs V, Luyckx K, Soenens B (2008) Reciprocal relations between teacher-child conflict and aggressive behavior in kindergarten: a three-wave longitudinal study. J Clin Child Adolesc Psychol 37:588-599. https://doi.org/10.1080/15374410802148079 
23. Webster-Stratton C (2001) The incredible years: parents, teachers, and children training series. Resid Treat Child Youth 18:31-45. https://doi.org/10.1300/J007v18n03_04

24. Webster-Stratton C (1994) The incredible years teacher training series. Incredible Years, Seattle, WA

25. Fossum S, Handegård BH, Drugli MB (2017) The incredible years teacher classroom management programme in Kindergartens: effects of a universal preventive effort. J Child Fam Stud 26:2215-2223. https://doi.org/10.1007/s10826-017-0727-3

26. Aasheim M, Reedtz C, Handegård BH, Martinussen M, Mørch W-T (2018) Evaluation of the incredible years teacher classroom management program in a regular Norwegian school setting. Scand J Educ Res:114. https://doi.org/10.1080/00313831.2018.1466357

27. Baker-Henningham H, Scott S, Jones K, Walker S (2012) Reducing child conduct problems and promoting social skills in a middle-income country: cluster randomised controlled trial. Br J Psychiatry 201:101-108. https://doi.org/10.1192/bjp.bp.111.096834

28. Raver CC, Jones SM, Li-Grining C, Zhai F, Metzger MW, Solomon B (2009) Targeting children's behavior problems in preschool classrooms: a cluster-randomized controlled trial. J Consult Clin Psychol 77:302-316. https://doi.org/10.1037/a0015302

29. Williford AP, Shelton TL (2008) Using mental health consultation to decrease disruptive behaviors in preschoolers: adapting an empirically-supported intervention. J Child Psychol Psychiatry 49:191-200. https://doi.org/10.1111/j.1469-7610.2007.01839.x

30. Carlson JS, Tiret HB, Bender SL, Benson L (2011) The influence of group training in the Incredible Years teacher classroom management program on preschool teachers' classroom management strategies. J Appl Sch Psychol 27:134-154. https://doi.org/10.1080/15377903.2011.565277

31. Shernoff ES, Kratochwill TR (2007) Transporting an evidence-based classroom management program for preschoolers with disruptive behavior problems to a school: an analysis of implementation, outcomes, and contextual variables. Sch Psychol Q 22:449-472. https://doi.org/10.1037/1045-3830.22.3.449

32. Statistics Norway (2018) Kindergartens - annually, final figures. https://www.ssb.no/en/utdanning/statistikker/barnehager/aar-endelige. Accessed: 28 Jun 2019.

33. Act no. 64 (2005) The Kindergarten Act. Oslo: Ministry of Education and Research. 
34. Achenbach TM, Rescorla LA (2000) Manual for the ASEBA preschool forms \& profiles: child behavior checklist for ages 1 1/2-5, language development survey, caregiver - teacher report form: an integrated system of multi-informant assessment. ASEBA, Burlington, VT

35. Snijders TAB, Bosker RJ (1999) Multilevel analysis: an introduction to basic and applied multilevel analysis. Sage, London, UK

36. Scherbaum CA, Ferreter JM (2009) Estimating statistical power and required sample sizes for organizational research using multilevel modeling. Organ Res Methods 12:347-367. https://doi.org/10.1177/1094428107308906

37. Lake S, Kammann E, Klar N, Betensky R (2002) Sample size re-estimation in cluster randomization trials. Stat Med 21:1337-1350. https://doi.org/10.1002/sim.1121

38. Webster-Stratton C (2011) Promoting positive academic and social behaviors. The teachers and children series. A video-based course (Lederveiledning - Norwegian edition). Incredible Years, Seattle, WA

39. Pianta RC (2001) Student-Teacher Relationship Scale: Professional Manual. Psychological Assessment Resources, Odessa, FL

40. Drugli MB, Hjemdal O (2013) Factor structure of the student-teacher relationship scale for Norwegian school-age children explored with confirmatory factor analysis. Scand J Educ Res 57:457-466. https://doi.org/10.1080/00313831.2012.656697

41. Tsigilis N, Gregoriadis A (2008) Measuring teacher-child relationships in the Greek kindergarten setting: a validity study of the Student-Teacher Relationship Scale-Short Form. Early Education and Development 19:816-835. https://doi.org/10.1080/10409280801975826

42. Kristensen S, Henriksen TB, Bilenberg N (2010) The Child Behavior Checklist for Ages 1.5-5 (CBCL/1 $1 \frac{1}{2-5}$ ): assessment and analysis of parent-and caregiver-reported problems in a population-based sample of Danish preschool children. Nord J Psychiatry 64:203-209. https://doi.org/10.3109/08039480903456595

43. Feingold A (2013) A regression framework for effect size assessments in longitudinal modeling of group differences. Rev Gen Psychol 17:111-121. https://doi.org/10.1037/a0030048 
44. Singer JD, Willett JB, Willett JB (2003) Applied longitudinal data analysis: modeling change and event occurrence. Oxford University Press, Oxford, UK. https://doi.org/10.1093/acprof:oso/9780195152968.001.0001

45. Cohen J (1988) Statistical power analysis for the behavioral sciences. 2nd edn. Laurence Erlbaum, Hillsdale, NJ

46. Rose G (1981) Strategy of prevention: lessons from cardiovascular disease. Br Med J (Clin Res Ed) 282:1847-1851. https://doi.org/10.1136/bmj.282.6279.1847

47. McLaren L, McIntyre L, Kirkpatrick S (2009) Rose's population strategy of prevention need not increase social inequalities in health. Int J Epidemiol 39:372-377. https://doi.org/10.1093/ije/dyp315

48. Henricsson L, Rydell A-M (2004) Elementary school children with behavior problems: teacher-child relations and self-perception. A prospective study. Merrill Palmer Q 50:111-138. https://doi.org/10.1353/mpq.2004.0012

49. Hamre BK, Pianta RC (2001) Early teacher-child relationships and the trajectory of children's school outcomes through eighth grade. Child Dev 72:625-638. https://doi.org/10.1111/1467-8624.00301

50. Neal JW, Durbin CE, Gornik AE, Lo SL (2017) Codevelopment of preschoolers' temperament traits and social play networks over an entire school year. J Pers Soc Psychol 113:627-640. https://doi.org/10.1037/pspp0000135

51. Drugli MB, Larsson B, Fossum S, Mørch WT (2010) Five-to six-year outcome and its prediction for children with ODD/CD treated with parent training. J Child Psychol Psychiatry 51:559-566. https://doi.org/10.1111/j.1469-7610.2009.02178.x

52. Reid MJ, Webster-Stratton C, Hammond M (2003) Follow-up of children who received the Incredible Years intervention for oppositional-defiant disorder: maintenance and prediction of 2-year outcome. Behav Ther 34:471-491. https://doi.org/10.1016/S0005-7894(03)80031-X

53. Raver CC, Jones SM, Li-Grining CP, Metzger M, Champion KM, Sardin L (2008) Improving preschool classroom processes: preliminary findings from a randomized trial implemented in head start settings. Early Child Res Q 23:10-26. https://doi.org/https://doi:10.1016/j.ecresq.2007.09.001 
54. Backer TE (2000) The failure of success: challenges of disseminating effective substance abuse prevention programs. J Community Psychol 28:363-373. https://doi.org/10.1002/(SICI)15206629(200005)28:3<363::AID-JCOP10>3.0.CO;2-T

55. Harris AD, McGregor JC, Perencevich EN, Furuno JP, Zhu J, Peterson DE, Finkelstein J (2006) The use and interpretation of quasi-experimental studies in medical informatics. J Am Med Inform Assoc 13:16-23. https://doi.org/10.1197/jamia.M1749

56. Shadish W, Cook TD, Campbell DT (2002) Experimental and quasi-experimental designs for generalized causal inference. Houghton Mifflin, Boston, MA

57. Ialongo NS, Werthamer L, Kellam SG, Brown CH, Wang S, Lin Y (1999) Proximal impact of two firstgrade preventive interventions on the early risk behaviors for later substance abuse, depression, and antisocial behavior. Am J Community Psychol 27:599-641. https://doi.org/10.1023/A:1022137920532 
Fig. 1 Flow diagram

\section{Enrolment}

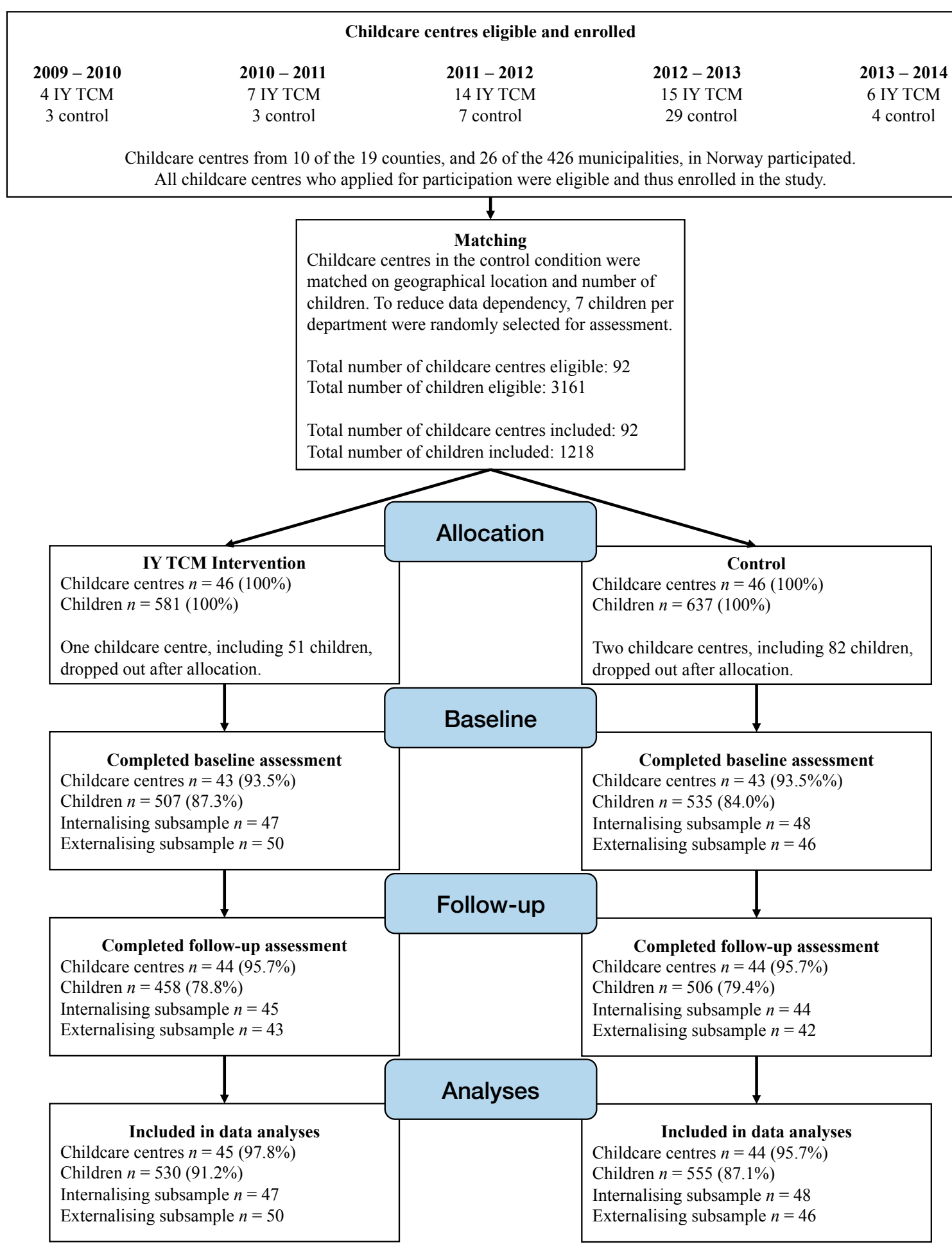


Fig. 2 Visualisation of the intervention effects on STRS-SF Closeness (top) and Conflict (bottom), for the entire sample (left), the internalising subsample (middle) and the externalising subsample (right). The results are based on estimated marginal means with standard errors from the linear mixed models
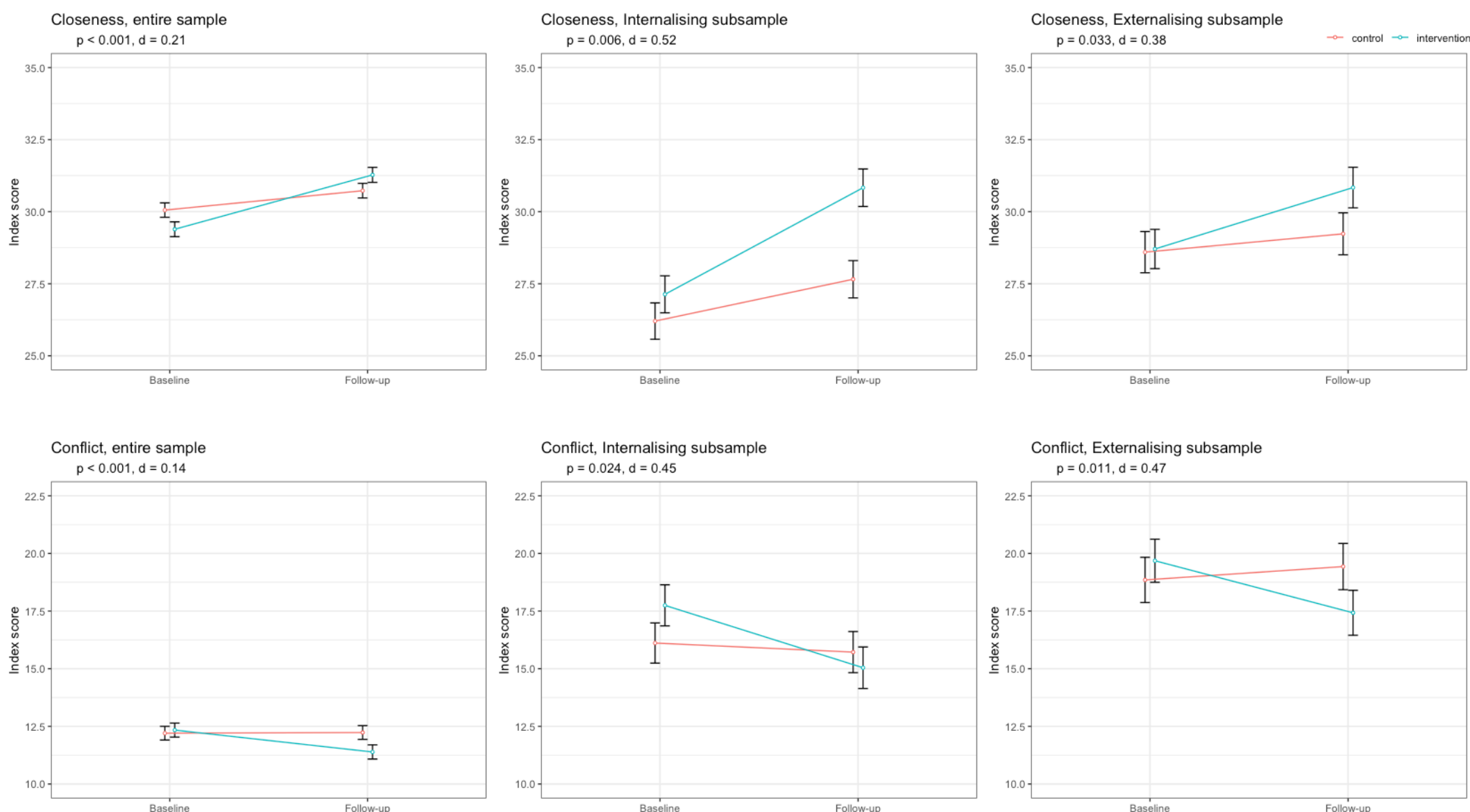


\section{Tables}

Table 1. Baseline characteristics

\begin{tabular}{|c|c|c|c|c|c|c|}
\hline & \multicolumn{2}{|c|}{ All participants } & \multicolumn{2}{|c|}{ Participants lost to follow-up } & \multicolumn{2}{|c|}{$\begin{array}{l}\text { Participants with data at both } \\
\text { timepoints }\end{array}$} \\
\hline & Intervention & Control & Intervention & Control & Intervention & Control \\
\hline \multicolumn{7}{|l|}{ Demographics } \\
\hline Number of children & 530 & 555 & 72 & 49 & 458 & 506 \\
\hline Females, n (\%) & $271(51.1)$ & $268(48.2)$ & $36(50.0)$ & $24(49.0)$ & $235(51.3)$ & $244(48.2)$ \\
\hline Years (age), mean $\pm \mathrm{SE}$ & $4.28 \pm 0.06$ & $4.15 \pm 0.06$ & $4.06 \pm 0.12$ & $4.31 \pm 0.14$ & $4.16 \pm 0.07$ & $4.27 \pm 0.06$ \\
\hline \multicolumn{7}{|l|}{ STRS-SF measures } \\
\hline Closeness, mean $\pm \mathrm{SE}$ & $30.04 \pm 0.28$ & $29.41 \pm 0.29$ & $29.15 \pm 0.57$ & $28.31 \pm 0.65$ & $29.46 \pm 0.30$ & $30.23 \pm 0.28$ \\
\hline Conflict, mean \pm SE & $12.19 \pm 0.31$ & $12.32 \pm 0.32$ & $13.00 \pm 0.68$ & $13.70 \pm 0.78$ & $12.20 \pm 0.34$ & $12.03 \pm 0.32$ \\
\hline \multicolumn{7}{|l|}{ C-TRF measures } \\
\hline Internalising, mean $\pm \mathrm{SE}$ & $3.27 \pm 0.27$ & $3.26 \pm 0.28$ & $3.85 \pm 0.57$ & $4.90 \pm 0.66$ & $3.16 \pm 0.29$ & $3.10 \pm 0.27$ \\
\hline Externalising, mean $\pm \mathrm{SE}$ & $4.83 \pm 0.47$ & $5.51 \pm 0.52$ & $7.66 \pm 1.62$ & $7.31 \pm 1.47$ & $5.35 \pm 0.53$ & $4.66 \pm 0.48$ \\
\hline \multicolumn{7}{|l|}{ High-risk subsamples } \\
\hline Internalising, $\mathrm{n}(\%)$ & $47(11.9)$ & $48(10.0)$ & $2(8.0)$ & $4(13.0)$ & $45(4.8)$ & $44(6.1)$ \\
\hline Externalising, $\mathrm{n}(\%)$ & $50(12.7)$ & $46(9.6)$ & $7(28.0)$ & $4(13.0)$ & $43(4.6)$ & $42(5.8)$ \\
\hline
\end{tabular}

Note: Continues variables are reported as estimated marginal means with standard errors from the linear mixed models. Group-by-attrition analyses revealed differences in STRS-SF Closeness, STRS-SF Conflict, and C-TRF Internalising between participants lost to follow-up and participants with data at both timepoints in the control condition. High-risk subsamples are defined as those that scored equal to or greater than the 90th percentile on the baseline C-TRF Internalising and Externalising scales and are based on valid C-TRF scores. 
Table 2. Estimated marginal means and standard errors at follow-up and estimates of change

\begin{tabular}{|c|c|c|c|c|c|c|c|c|c|c|c|c|c|}
\hline & \multicolumn{4}{|c|}{ Intervention } & \multicolumn{4}{|c|}{ Control } & & & & & \\
\hline & \multicolumn{2}{|c|}{ Baseline } & \multicolumn{2}{|c|}{ Follow-up } & \multicolumn{2}{|c|}{ Baseline } & \multicolumn{2}{|c|}{ Follow-up } & \multicolumn{2}{|c|}{ Group difference at baseline } & \multicolumn{3}{|c|}{ Time* group difference estimate } \\
\hline & $N$ & mean $\pm S E$ & $N$ & mean $\pm S E$ & $N$ & mean $\pm S E$ & $N$ & mean $\pm S E$ & Estimate $(95 \% \mathrm{CI})$ & $p$ & Estimate $(95 \% \mathrm{CI})$ & $p$ & $d$ \\
\hline \multicolumn{14}{|l|}{ Closeness } \\
\hline Entire sample & 507 & $29.39 \pm 0.26$ & 458 & $31.28 \pm 0.26$ & 535 & $30.06 \pm 0.25$ & 506 & $30.73 \pm 0.25$ & $0.67(-0.04$ to 1.37$)$ & 0.065 & $1.21(0.78$ to 1.65$)$ & $<0.001$ & 0.21 \\
\hline Internalising sample & 47 & $27.10 \pm 0.63$ & 45 & $30.79 \pm 0.64$ & 48 & $26.24 \pm 0.62$ & 44 & $27.70 \pm 0.64$ & $0.86(-0.87$ to 2.59$)$ & 0.334 & 2.24 ( 0.69 to 3.78$)$ & 0.006 & 0.52 \\
\hline Externalising sample & 50 & $28.78 \pm 0.68$ & 43 & $30.97 \pm 0.61$ & 46 & $28.71 \pm 0.60$ & 42 & $29.33 \pm 0.62$ & $0.08(-1.57$ to 1.72$)$ & 0.929 & $1.56(0.15$ to 2.98$)$ & 0.033 & 0.38 \\
\hline \multicolumn{14}{|l|}{ Conflict } \\
\hline Entire sample & 507 & $12.34 \pm 0.30$ & 458 & $11.39 \pm 0.30$ & 535 & $12.21 \pm 0.30$ & 506 & $12.23 \pm 0.30$ & $-0.13(-0.70$ to 0.96$)$ & 0.752 & $-0.97(-1.49$ to -0.46$)$ & $<0.001$ & 0.14 \\
\hline Internalising sample & 47 & $17.49 \pm 0.75$ & 45 & $14.80 \pm 0.77$ & 48 & $16.28 \pm 0.75$ & 44 & $15.91 \pm 0.77$ & $1.21(-0.86$ to 3.30$)$ & 0.253 & $-2.33(-4.33$ to -0.34$)$ & 0.024 & 0.45 \\
\hline Externalising sample & 50 & $19.62 \pm 0.87$ & 43 & $17.30 \pm 0.91$ & 46 & $18.70 \pm 0.91$ & 42 & $19.31 \pm 0.93$ & $0.92(-1.54$ to 3.80$)$ & 0.465 & $-2.93(-5.15$ to -0.71$)$ & 0.011 & 0.47 \\
\hline
\end{tabular}

Note: Results are reported as estimated marginal means with standard errors from the linear mixed models. High-risk subsamples are defined as those that scored equal to or greater than the 90th percentile on the baseline C-TRF Internalising and Externalising scales and are based on valid C-TRF scores. For both high-risk subsamples, comorbidity was controlled for. $d=$ effect sizes were computed as the estimate of the group*time effect divided by the pooled standard deviation at baseline. ICC for STRS-SF Closeness at baseline $=0.38$, ICC for STRS-SF Conflict at baseline $=0.34$. 